\title{
Chaotic Inflation in Supergravity with Heisenberg Symmetry
}

\author{
Stefan Antusch, ${ }^{1}$ Mar Bastero-Gil,${ }^{2}$ Koushik Dutta, ${ }^{1}$ Steve F. King, ${ }^{3}$ and Philipp M. Kostka ${ }^{1}$ \\ ${ }^{1}$ Max-Planck-Institut für Physik (Werner-Heisenberg-Institut), \\ Föhringer Ring 6, 80805 München, Germany \\ ${ }^{2}$ Departamento de Fisica Teorica y del Cosmos and \\ Centro Andaluz de Fisica de Particulas Elementales, \\ Universidad de Granada, 19071 Granada, Spain \\ ${ }^{3}$ School of Physics and Astronomy, University of Southampton, \\ Southampton, SO17 1BJ, United Kingdom
}

\begin{abstract}
We propose the introduction of a Heisenberg symmetry of the Kähler potential to solve the problems with chaotic inflation in supergravity, as a viable alternative to the use of shift symmetry. The slope of the inflaton potential emerges from a small Heisenberg symmetry breaking term in the superpotential. The modulus field of the Heisenberg symmetry is stabilized and made heavy with the help of the large vacuum energy density during inflation. The observable predictions are indistinguishable from those of typical chaotic inflation models, however the form of the inflationary superpotential considered here may be interpreted in terms of sneutrino inflation arising from certain classes of string theory.
\end{abstract}

\section{INTRODUCTION}

The paradigm of inflation at the beginning of the evolution of the Universe is very successful in explaining why the observable Universe is homogeneous and isotropic on the largest scales, yet with the structures on small scales [1]. Among several models of inflation, chaotic inflation is very attractive, mainly due to its simplistic form of the potential and the associated dynamics of the inflaton field [2]. On the other hand, successful realization of chaotic inflation requires field values which are superPlanckian. From the observational point of view, chaotic inflation is distinguished from small field inflation models by its prediction of larger tensor fluctuations [3].

A generic problem of inflation models, when embedded in supergravity (SUGRA) is that typically a large mass for the inflaton field of the order of the Hubble scale during inflation (which implies a slow-roll parameter $\eta \sim 1$ ) is generated and spoils inflation. This is called the $\eta$ problem in SUGRA inflation [4]. The problem arises from the SUGRA potential which generally contains a term of the form $e^{\left(K / M_{P}^{2}\right)} V_{0}$, where $V_{0}$ is the vacuum energy during inflation (up to a constant). For example, assuming a minimal canonical Kähler potential $K$, this becomes $e^{\left(\phi \phi^{*} / M_{P}^{2}\right)} V_{0}$, which, when expanded in powers of $\phi \phi^{*}$, where $\phi$ is the inflaton field, leads to a large mass term of order the Hubble constant. The problem is particularly acute in chaotic inflation in which the inflaton field value exceeds the Planck mass $M_{P}$.

The above problem of chaotic inflation can be alleviated by assuming some particular non-minimal form of the Kähler potential, however many of the proposed forms are not enforced by any symmetry [5] and require some amount of tuning of parameters. A more promising approach is to impose a symmetry on the Kähler function such that the inflaton field does not explicitly appear in the Kähler potential, thereby finessing the problem of not allowing super-Planckian field values arising from the exponential in the potential. For example, imposition of a shift symmetry makes the Kähler potential independent of the real component of the complex chiral superfield $\Phi$, thus allowing this direction to be identified as the inflaton field [6].

In a recent paper [7] we proposed a class of models in which the $\eta$-problem of supersymmetric hybrid inflation may be resolved using a Heisenberg symmetry. For example, Heisenberg symmetry includes the case of no-scale supergravity Kähler potential, which is endemic to string theory. Due to the nature of the symmetry, the inflaton $\Phi$ appears in the Kähler potential only as a combination of $\rho=T+T^{*}-|\Phi|^{2}$, where $T$ is a modulus field $[8,9]$. In this approach the associated modulus field is stabilized and made heavy with the help of the large vacuum energy during inflation without any fine-tuning. Because of the Heisenberg symmetry of the Kähler potential, the treelevel potential of the inflaton is flat and only lifted by radiative corrections, induced by Heisenberg symmetry breaking superpotential couplings.

In this letter we propose the introduction of a Heisenberg symmetry of the Kähler potential to solve the problems with chaotic inflation in SUGRA. As inflationary part of the superpotential we will consider $W=M \Phi X$, where $M$ is a mass parameter, $\Phi$ is the superfield containing the inflaton and $X$ is an auxiliary superfield. ${ }^{1}$ This form of the superpotential has been discussed for chaotic inflation in [6], where a shift symmetry has been used

\footnotetext{
${ }^{1}$ We require the chaotic superpotential to be of this form, rather than the more common form $W=M \Phi^{2}$ since, according to our mechanism, the field $X$ will quickly settle to a zero value during inflation, resulting in the superpotential also being zero during inflation, which is a crucial requirement for solving the $\eta$ problem in this approach.
} 
to protect the flatness of the inflaton potential. Using the Heisenberg symmetry with this form of the superpotential, we show that the $\eta$-problem of SUGRA inflation is resolved, allowing well behaved inflaton field values larger than the Planck mass, as required for a successful realization of chaotic inflation. We also show that, as in the case of hybrid inflation [7], the associated modulus field will be stabilized during inflation by the large vacuum energy. It turns out that the dynamics of the model will be effectively governed by a single scalar field with a tree-level potential which has a purely quadratic dependence on the inflaton field. (We estimate the effects of quantum corrections and show that they are negligible.) Thus we find that the observable predictions for the minimal setup discussed in this paper are indistinguishable from those of typical chaotic inflation models. However, the framework proposed here will have important implications when the theory of inflation is embedded into a particle physics model.

The paper is organized as follows: In the next section we present the framework of our model. In section 3, we discuss the effective tree-level scalar potential. The dynamics of all the scalar fields is discussed in detail in section 4 . In section 5 we summarize and conclude. In the Appendix we show that the quantum loop corrections do not change the tree-level potential.

\section{FRAMEWORK}

To implement chaotic inflation in SUGRA using Heisenberg symmetry we propose the following superpotential and Kähler potential,

$$
\begin{aligned}
W & =M \Phi X \\
K & \equiv\left(1+\kappa_{X}|X|^{2}+\kappa_{\rho} \rho\right)|X|^{2}+f(\rho) .
\end{aligned}
$$

In the Kähler potential the modulus $T$ together with the chiral inflaton superfield $\Phi$ appear only in the Heisenberg symmetric combination [8]

$$
\rho=T+T^{*}-|\Phi|^{2} .
$$

Here $X$ is an additional superfield that is supposed to be fixed at zero when inflation is operative. The nonzero $\mathrm{F}$-term from $X$ contributes to the vacuum energy required for inflation. Therefore, we realize inflation in this framework with vanishing inflationary part of the superpotential. We note that the superpotential of the form of Eq. (1) breaks the Heisenberg symmetry of the Kähler potential in Eq. (2) and gives rise to a $M^{2} \phi^{2}$ potential for the scalar component of $\Phi$ at tree-level. This model is 'natural' in the sense that setting the small breaking parameter to zero allows us to realize enhanced symmetry [10]. We take all chiral superfields to be gauge singlets, such that D-term contributions to the potential are absent.

\section{SCALAR POTENTIAL}

A Heisenberg symmetry of the Kähler potential allows us to implement super-Planckian values for the inflaton field in SUGRA theories, as can be seen by looking at the full (F-term) scalar potential given by ${ }^{2}$

$$
V_{\mathrm{F}}=\mathrm{e}^{K}\left[K^{i \bar{j}} \mathcal{D}_{i} W \mathcal{D}_{\bar{j}} W^{*}-3|W|^{2}\right],
$$

where the definition

$$
\mathcal{D}_{i} W:=W_{i}+K_{i} W
$$

has been used. The lower indices $i, j$ on the superpotential or Kähler potential denote the derivatives with respect to the chiral superfields or their conjugate (where a bar is involved). The inverse Kähler metric is defined as $K^{i \bar{j}}=K_{i \bar{j}}^{-1}$. Due to the Heisenberg symmetry of the Kähler potential the exponential in Eq. (4) is independent of the inflaton field and therefore we can realize field values larger than $M_{P}$ as required for chaotic inflation.

We will show below that the mass of the $X$ field is very large compared to the Hubble scale due to the $\kappa_{X}$ coupling in the Kähler potential and it settles to its minimum at $X=0$ very quickly before inflation. This essentially makes the superpotential $W$ of Eq. (1) vanish during inflation. An attractive feature of having $W=0$ during inflation is that it typically cancels several couplings between the inflaton sector and any other possibly existing scalar field sector in the theory ${ }^{3}$.

The Kähler metric can be calculated as the second derivatives of the Kähler potential in Eq. (2) with respect to the superfields and their conjugates, and along the direction $X=0$ in $(X, \Phi, T)$-basis this reduces to the block-diagonal form

$$
\left(K_{i \bar{j}}\right)=\left(\begin{array}{ccc}
1+\kappa_{\rho} \rho & 0 & 0 \\
0 & f^{\prime \prime}(\rho)|\phi|^{2}-f^{\prime}(\rho) & -f^{\prime \prime}(\rho) \phi^{*} \\
0 & -f^{\prime \prime}(\rho) \phi & f^{\prime \prime}(\rho)
\end{array}\right) .
$$

Here $\phi$ denotes the complex scalar component of the inflaton superfield $\Phi$. For $X=0$ the potential reduces to

$$
V_{\mathrm{F}}=e^{K} K^{X \bar{X}}\left|W_{X}\right|^{2} .
$$

Now we make a particular choice of $f(\rho)$ of the no-scale form 12

$$
f(\rho)=-3 \ln \rho .
$$

It provides a simple example of a Kähler potential invariant under the Heisenberg symmetry and arises naturally

\footnotetext{
2 Here we use a convention in which we set the reduced Planck mass $M_{\mathrm{P}} \simeq 2.4 \times 10^{18} \mathrm{GeV}$ to unity.

${ }^{3}$ In the context of Hybrid inflation with shift symmetry, see Ref. 11].
} 
in orbifold compactifications of heterotic string models [13]. We would like to remark that for our approach to work this specific form of the Kähler potential is not required, however it is well motivated from string theory and serves well to illustrate the modulus stabilization mechanism through the coupling with $X$ in the Kähler potential. After inflation, when $X \sim \Phi \sim 0$, another sector of the model will be responsible for SUSY breaking which may lead, for instance, to an effective no-scale model with radiatively induced gravitino mass.

With $f(\rho)$ as in Eq. (8), the potential reduces to the simple form

$$
V_{\mathrm{F}}=\frac{M^{2}|\phi|^{2}}{\rho^{3}\left(1+\kappa_{\rho} \rho\right)} .
$$

For any fixed value of $\rho$ the potential is just a mass term for the $\phi$ field that is suitable for chaotic inflation. This potential has a minimum at ${ }^{4}$

$$
\rho_{0}=-\frac{3}{4 \kappa_{\rho}} .
$$

Considering that $\rho$ is always positive, $\kappa_{\rho}$ must be negative and, as an example, we will choose its value to be $\kappa_{\rho}=-1$. Other than that, the form of the potential has a pole at the value of $\rho=-1 / \kappa_{\rho}$ and for values $\rho>-1 / \kappa_{\rho}$ the potential is negative and has a runaway behavior. Therefore, inflation happens only in the range of field values $0<\rho<-1 / \kappa_{\rho}$ and we assume it for all our considerations.

At the start of inflation $m_{\rho}^{2} \sim\left|W_{X}\right|^{2}$ and the squared Hubble scale is also of the same order. On the other hand $m_{\phi} \sim M \ll H$ during inflation. Therefore the $\rho$ field settles to its minimum very quickly whereas the inflaton field, being light, slow-rolls along its potential. When $\rho$ has settled to its minimum and $\phi$ is slowly rolling the vacuum energy dominates and drives inflation. In fact, the coupling $\kappa_{\rho}$ between $\rho$ and $X$ in the Kähler potential induces a mass for the $\rho$ field of the order of the vacuum energy during inflation and it allows the modulus to be stabilized very quickly before inflation. For $\kappa_{\rho}=0$ in the expression for the scalar potential of Eq. (9), we can see that the $\rho$ field would have a runaway potential. The role of this coupling in the context of hybrid inflation with Heisenberg symmetry was first mentioned in Ref. 7]. Although $\rho_{0}$ is independent of $\phi$, the field $\rho$ is not absolutely fixed at the minimum of the potential due to the presence of effects from non-canonical kinetic terms. We will verify these qualitative statements in the next section with full evolution equations of the fields. We also note that when $X$ and $\rho$ settle to their respective minima (i.e. $X=0$ and $\rho=\rho_{0}$ ) the potential has the same form as the corresponding global SUSY potential.

\footnotetext{
${ }^{4}$ For a general function $f(\rho)$, the minimum is given by $f^{\prime}\left(\rho_{0}\right)\left(1+\kappa_{\rho} \rho_{0}\right)=\kappa_{\rho}$.
}

For example in our case, once the modulus is stabilized at the minimum during inflation, the tree-level potential reduces to a quadratic potential, as can be seen from Eq. (9). We will discuss the scalar mass spectrum in the following section.

\section{DYNAMICS OF THE FIELDS}

In the previous section we have discussed the form of the potential when the $X$ field has settled to its minimum and argued qualitatively that the $\rho$ field will also get stabilized quickly such that successful chaotic inflation can be realized. In this section we calculate the masses of the fields and perform a full numerical simulation to investigate the dynamics of the fields.

We begin with writing down the kinetic energy terms for the fields. With $X=X^{*}=0$ in the Kähler metric, the kinetic terms are given by:

$\mathcal{L}_{\text {kin }}=\left(1+\kappa_{\rho} \rho\right)\left|\partial_{\mu} X\right|^{2}+\frac{3}{\rho}\left|\partial_{\mu} \phi\right|^{2}+\frac{3}{4 \rho^{2}}\left(\partial_{\mu} \rho\right)^{2}+\frac{3}{4 \rho^{2}}\left(I_{\mu}\right)^{2}$,

where

$$
I_{\mu}=\mathrm{i}\left(\partial_{\mu}\left(T-T^{*}\right)+\phi \partial_{\mu} \phi^{*}-\phi^{*} \partial_{\mu} \phi\right) .
$$

Since the phases of the scalar fields $\phi, X$ as well as $\operatorname{Im}(T)$ very quickly approach a constant value in an expanding Universe and subsequently decouple from the absolute values and $\operatorname{Re}(T)$ in the equations of motion (as has been discussed in the Appendix of [7]), we only consider the absolute values $\varphi=\sqrt{2}|\phi|, x=\sqrt{2}|X|$ and the real field $\rho$ in what follows. In this case, $I_{\mu}$ vanishes identically.

The evolution equations for the background field values $\rho, \varphi$ and $x$ in an expanding universe are then given by:

$$
\begin{array}{r}
\ddot{x}+3 H \dot{x}+\frac{1}{\left(1+\kappa_{\rho} \rho\right)}\left(\kappa_{\rho} \dot{\rho} \dot{x}+V_{x}\right)=0, \\
\ddot{\varphi}+3 H \dot{\varphi}-\frac{1}{\rho} \dot{\rho} \dot{\varphi}+\frac{\rho}{3} V_{\varphi}=0, \\
\ddot{\rho}+3 H \dot{\rho}-\frac{1}{\rho} \dot{\rho}^{2}+\dot{\varphi}^{2}-\frac{\kappa_{\rho}}{3} \rho^{2} \dot{x}+\frac{2}{3} \rho^{2} V_{\rho}=0,
\end{array}
$$

where $V_{\rho}, V_{\varphi}, V_{x}$ are the derivatives of the potential with respect to the fields, and $H$ is the Hubble expansion rate. For large enough values of $\varphi$, the fields follow a slow-roll trajectory with $\rho$ being practically at the minimum of the potential.

In Fig. 1 we show the dynamics of the fields for generic initial conditions. As one can see, the $x$ field settles to its minimum followed by the $\rho$ field, whereas the inflaton field remains slowly rolling for more than 60 e-folds of inflation. As the inflaton field rolls down towards its minimum, the vacuum energy decreases and thus also the mass of the $\rho$ field. When the slow-roll conditions are violated the inflaton field acquires a large velocity and it provides a kick to the evolution of $\rho$ due to the $\dot{\varphi}^{2}$ term in its equation of motion. At the end of inflation the $\varphi$ 
field starts oscillating, the velocity term gets damped and finally the $\rho$ field settles to a slightly different field value. However, at this epoch we expect some other modulistabilization mechanism to start playing a role.

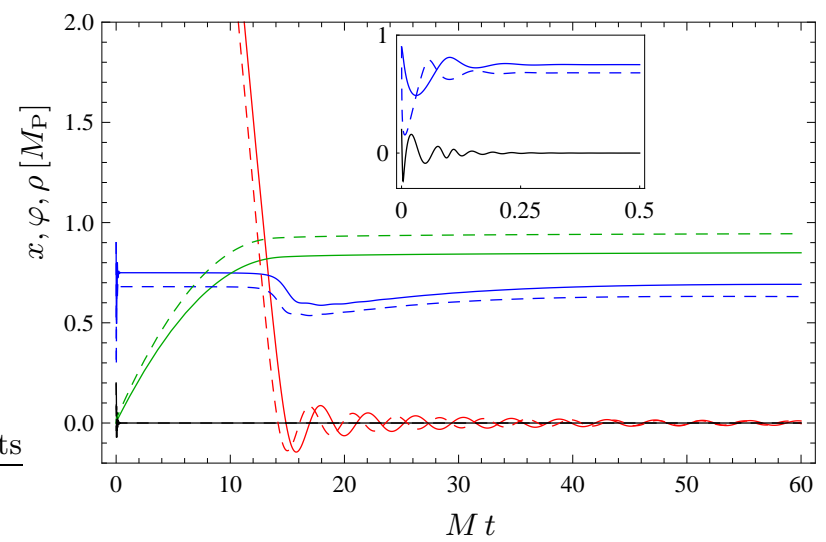

FIG. 1: Evolution of the fields. The green line represents the number of e-folds $/ 100$, the red line represents the inflaton direction $\varphi$, the blue line depicts the evolution of $\rho$ and the black line the evolution of $x$. Dashed lines represent the evolution of the same fields if in addition to the tree-level potential, quantum corrections are taken into account (see Appendix).

In the following, we denote as physical fermions and scalars those with canonical kinetic terms. In terms of the original fields $\Psi_{i}=\{X, \phi, T\}$, the Lagrangian for the scalar kinetic terms reads:

$$
\mathcal{L}_{\text {kin }}=K_{i j *} \partial_{\mu} \Psi_{i} \partial^{\mu} \Psi_{j}^{*},
$$

and similarly for the fermions. We can canonically normalize the fields by expanding the Lagrangian around the minimum. In particular, during inflation we have $X=0$, $\rho \simeq \rho_{0}=-3 / 4 \kappa_{\rho}$, and $\phi$ changing slowly with time. The field $\rho$ can be redefined as canonically normalized field $\tilde{\rho}$ by:

$$
\tilde{\rho}=\sqrt{\frac{3}{2}} \ln \rho .
$$

The remaining non-canonical factors in Eq. (11) only depend on $\rho$ (or $\tilde{\rho}$ ), and we can define the physical states during inflation by expanding those factors around $\rho_{0}$ :

$$
\mathcal{L}_{\text {kin }}=\left(1+\kappa_{\rho} \rho_{0}\right)\left|\partial_{\mu} X\right|^{2}+\frac{3}{\rho_{0}}\left|\partial_{\mu} \phi\right|^{2}+\frac{1}{2}\left(\partial_{\mu} \tilde{\rho}\right)^{2}+\cdots .
$$

Then, with normalization factors $\left(1+\kappa_{\rho} \rho_{0}, 3 / \rho_{0}, 1\right)$, for $(\tilde{x}, \tilde{\varphi}, \tilde{\rho})$, the physical squared scalar masses are given by:

$$
\begin{aligned}
& m_{\tilde{x}}^{2}=\frac{64}{27} \kappa_{\rho}^{2} M^{2}\left(1+32 \kappa_{X} \kappa_{\rho} \tilde{\varphi}^{2}\right), \\
& m_{\tilde{\varphi}}^{2}=-\frac{64}{27} \kappa_{\rho}^{3} M^{2} \\
& m_{\tilde{\rho}}^{2}=-\frac{256}{27} \kappa_{\rho}^{3} M^{2} \tilde{\varphi}^{2} .
\end{aligned}
$$

Fields with a tilde are canonically normalized. To calculate their masses, we have assumed that $\rho$ has settled to its minimum (where $\kappa_{\rho}$ has been left general instead of setting it to -1 as done before). First we note that the canonically normalized inflaton field has a mass smaller than the Hubble scale during inflation where the field value is super-Planckian. For $\kappa_{X}$ negative, the mass of the $\tilde{x}$ field is larger than the mass of $\tilde{\rho}$ and both masses are larger than the Hubble scale $H \sim \sqrt{V / 3}$. Therefore, as we have shown in the numerical simulations, the $x$ field settles quickly to its minimum $x=0$, followed by the $\rho$ field.

Similar to the conventional chaotic inflation model with purely quadratic potential the prediction for the spectral index $n_{s}$ and the tensor-to-scalar ratio $r$ are given by

$$
\begin{aligned}
n_{s} & \simeq 1-2 / N, \\
r & \simeq 8 / N,
\end{aligned}
$$

where $N$ is the number of e-folds before the end of inflation where the observable scales have crossed the horizon. For 60 e-folds of inflation the predicted value of the spectral index is $n_{s} \sim 0.97$, well consistent with all available cosmological data [14]. In addition, the tensor-to-scalar ratio is predicted as $r \sim 0.13$ (for $N=60$ ), which might be probed by the upcoming PLANCK satellite [15].

\section{CONCLUSIONS}

We have proposed the introduction of a Heisenberg symmetry of the Kähler potential to solve the problems with chaotic inflation in SUGRA, as a viable alternative to the use of shift symmetry. We have focussed on a particular form of chaotic inflation based on the superpotential $W=M \Phi X$, where $M$ is a Heisenberg symmetry breaking mass parameter, $\Phi$ is the superfield which contains the inflaton and $X$ is an auxiliary superfield. Within this framework we have shown that the $\eta$ - problem of the SUGRA potential is resolved, allowing well behaved inflaton field values larger than the Planck mass, as required for a successful realization of chaotic inflation. We have also shown that the associated modulus field is stabilized during inflation by the large vacuum energy.

We have seen that the dynamics of the model is governed by a single scalar field with a tree-level potential which has a purely quadratic dependence on the inflaton field. In an Appendix we have estimated the effects of quantum corrections and shown that they are negligible. Thus we find that the observable predictions for the minimal setup discussed in this paper are indistinguishable from those of typical chaotic inflation models, namely a spectral index $n_{s} \sim 0.97$ and a tensor-to-scalar ratio $r \sim 0.13$.

However the framework proposed here will have important implications when the theory of inflation is embedded into a particle physics model. For example, the effective mass of the inflaton differs from the mass scale $M$ by 
a factor which depends on $\kappa_{\rho}$ (cf. Eq. (18)). If the present chaotic inflation model is interpreted as a (right-handed) sneutrino inflation model [16], then this would change the mass scale of the associated right-handed neutrinos. It is also worth remarking that the type of chaotic superpotential considered here would correspond to Dirac right-handed neutrino masses of the form $M N_{1} N_{2}$ where $N_{1}$ is identified with $\Phi$ and $N_{2}$ is identified with $X$. Such a Dirac right-handed neutrino mass structure is motivated by certain classes of Heterotic string theory [17]. It would be interesting to discuss such a Dirac sneutrino interpretation of the inflation model considered here, and the associated implications for leptogenesis, however this would require an analysis of the evolution of the $\rho$ field after inflation which is beyond the scope of this letter. The embedding into a particle physics model would also allow to address the issue of reheating. For instance, in sneutrino inflation, the inflaton would decay via its neutrino Yukawa couplings.

In conclusion, we have proposed the introduction of a Heisenberg symmetry of the Kähler potential to solve the problems with chaotic inflation in SUGRA, as a viable alternative to the use of shift symmetry. It leads to the same predictions for $n_{s}$ and $r$, however has different implications when embedded into a particle physics model.

\section{Appendix: Calculations of Quantum Loop Corrections}

Here we discuss that the quantum corrections to the potential have negligible effects. We therefore calculate the one-loop effective potential from the formulae given in [9, 18]. Introducing a cutoff $\Lambda=M_{\mathrm{P}}$ in the theory, the one-loop correction to the effective potential is given by

$$
V_{\text {loop }}=\frac{\Lambda^{2}}{32 \pi^{2}} \operatorname{Str} \mathcal{M}^{2}+\frac{1}{64 \pi^{2}} \operatorname{Str} \mathcal{M}^{4} \ln \left(\frac{\mathcal{M}^{2}}{\Lambda^{2}}\right) .
$$

Since in order to fit observations, the mass parameter $M$ should be of the order $M \sim \mathcal{O}\left(10^{-5}\right)$, we can safely ignore the logarithmic part of the loop-correction.

For the dominant quadratic part, the supertrace can be written in the simple form

$$
\begin{aligned}
\operatorname{Str} \mathcal{M}^{2}= & 2\left(N_{\text {tot }}-1\right) V \\
& +2|W|^{2} \mathrm{e}^{K}\left(N_{\text {tot }}-1-G^{i} R_{i \bar{j}} G^{\bar{j}}\right),
\end{aligned}
$$

where $N_{\text {tot }}=3$ is the total number of chiral superfields and the Kähler function reads

$$
G=K+\ln |W|^{2} .
$$

The contribution due to the Ricci tensor of the Kähler manifold in Eq. (23) is the following

$$
R_{i \bar{j}}=\partial_{i} \partial_{\bar{j}} \ln \operatorname{det}\left(G_{m \bar{n}}\right) .
$$

Taking $\kappa_{X}<0$, the curvature along the $X$-direction is large and positive during inflation when $\varphi \neq 0$, so that the field will quickly go to zero. For the sake of simplicity, we set $x=0$ which is justified by the simulation with the full $x$-dependent potential as depicted in Fig. 11. Plugging the superpotential and Kähler potential of Eqs. (1), (2) into Eq. (23), we end up with the $\rho$ - and $\varphi$-dependent loop-potential

$$
V_{\text {loop }}=\frac{M^{2} \varphi^{2}}{32 \pi^{2}\left(3 \rho^{3}\right)}\left[\frac{2\left(3+4 \kappa_{\rho} \rho\right)}{\left(1+\kappa_{\rho} \rho\right)^{2}}-\frac{\left(\kappa_{\rho} \rho+12 \kappa_{X}\right)}{\left(1+\kappa_{\rho} \rho\right)^{3}}\right] .
$$

Therefore, the presence of loop-corrections just has the effect of shifting the minimum of the potential in the $\rho$-direction (cf. Fig. 11). For example with $\kappa_{\rho}=-1$ and $\kappa_{X}=-1$, the loop-corrected minimum shifts to $\rho_{0}=0.68$ from its tree-level value of $\rho_{0}=0.75$. It is important to note that similar to the tree-level potential, the loopcorrected potential also has a $\varphi$-independent minimum $\rho_{0}$. Once the $\rho$-field gets stabilized in its new minimum, this gives a different factor in front of the mass squared. In order to fit the amplitude of the curvature perturbation $P_{\mathcal{R}}$, one simply absorbs this factor in $M^{2}$ and adjusts the new effective mass squared. The amplitude of the primordial spectrum is given by $P_{\mathcal{R}}^{1 / 2} \simeq(N / \sqrt{6} \pi) m_{\text {eff }}$, and the WMAP normalization by $P_{\mathcal{R}}^{1 / 2} \simeq 5 \times 10^{-5}$ then gives $m_{\text {eff }} \simeq 6 \times 10^{-6}$. We therefore conclude that the loopcorrections do not change the predictions calculated from the tree-level potential but instead just lead to a massrenormalization of the inflaton field.

\section{Acknowledgments}

S.F.K. acknowledges partial support from the following grants: STFC Rolling Grant ST/G000557/1; EU Network MRTN-CT-2004-503369; EU ILIAS RII3-CT-2004506222. S.A., K.D. and P.M.K. were partially supported by the the DFG cluster of excellence "Origin and Structure of the Universe". The work of M.B.G. is partially supported by the M.E.C. grant FIS 2007-63364 and by the Junta de Andalucía group FQM 101.
[1] For textbook reviews on inflation see: A. R. Liddle and D. H. Lyth, "Cosmological inflation and large-scale structure," Cambridge, UK: Univ. Pr. (2000) 400 p;
A. D. Linde, "Particle Physics and Inflationary Cosmology," arXiv:hep-th/0503203; V. Mukhanov, "Physical Foundations of Cosmology," Cambridge, UK: Univ. Pr. 
(2005) $421 p$

[2] A. D. Linde, "Chaotic Inflation," Phys. Lett. B 129, 177 (1983).

[3] D. H. Lyth, "What would we learn by detecting a gravitational wave signal in the cosmic microwave background anisotropy?," Phys. Rev. Lett. 78, 1861 (1997) arXiv:hep-ph/9606387.

[4] E. J. Copeland, A. R. Liddle, D. H. Lyth, E. D. Stewart and D. Wands, "False vacuum inflation with Einstein gravity," Phys. Rev. D 49, 6410 (1994) arXiv:astro-ph/9401011]; A. D. Linde and A. Riotto, "Hybrid inflation in supergravity," Phys. Rev. D 56, 1841 (1997) arXiv:hep-ph/9703209; M. Dine, L. Randall and S. D. Thomas, "Supersymmetry breaking in the early universe," Phys. Rev. Lett. 75, 398 (1995) arXiv:hep-ph/9503303.

[5] A. B. Goncharov and A. D. Linde, "Chaotic Inflation In Supergravity," Phys. Lett. B 139, 27 (1984); H. Murayama, H. Suzuki, T. Yanagida and J. Yokoyama, "Chaotic inflation and baryogenesis in supergravity," Phys. Rev. D 50, 2356 (1994) arXiv:hep-ph/9311326.

[6] M. Kawasaki, M. Yamaguchi and T. Yanagida, "Natural chaotic inflation in supergravity," Phys. Rev. Lett. 85, 3572 (2000) arXiv:hep-ph/0004243; M. Kawasaki, M. Yamaguchi and T. Yanagida, "Natural chaotic inflation in supergravity and leptogenesis," Phys. Rev. D 63, 103514 (2001) arXiv:hep-ph/0011104.

[7] S. Antusch, M. Bastero-Gil, K. Dutta, S. F. King and P. M. Kostka, "Solving the $\eta$-Problem in Hybrid Inflation with Heisenberg Symmetry and Stabilized Modulus," JCAP 0901, 040 (2009) arXiv:0808.2425 [hep-ph]].

[8] P. Binetruy and M. K. Gaillard, "Noncompact symmetries and scalar masses is superstring inspired models." Phys. Lett. B 195, 382 (1987).

[9] M. K. Gaillard, H. Murayama and K. A. Olive, "Preserving flat directions during inflation," Phys. Lett. B 355, 71 (1995) arXiv:hep-ph/9504307.

[10] G. 't Hooft in "Recent Developments In Gauge Theories. Proceedings, Nato Advanced Study Institute, Cargese, France, August 26 - September 8, 1979," New York, Usa: Plenum (1980)

[11] S. Antusch, K. Dutta and P. M. Kostka, "SUGRA Hybrid Inflation with Shift Symmetry," Phys. Lett. B 677, 221 (2009) arXiv:0902.2934 [hep-ph]].
[12] E. Cremmer, S. Ferrara, C. Kounnas and D. V. Nanopoulos, "Naturally Vanishing Cosmological Constant In N=1 Supergravity," Phys. Lett. B 133, 61 (1983); for a review see: A. B. Lahanas and D. V. Nanopoulos, "The Road to No Scale Supergravity," Phys. Rept. 145, 1 (1987).

[13] E. Witten, "Dimensional Reduction Of Superstring Models," Phys. Lett. B 155, 151 (1985); S. Ferrara, C. Kounnas and M. Porrati, "General Dimensional Reduction Of Ten-Dimensional Supergravity And Superstring," Phys. Lett. B 181, 263 (1986); S. Ferrara, .. D. Lust and S. Theisen, "Target Space Modular Invariance and LowEnergy Couplings in Orbifold Compactifications," Phys. Lett. B 233, 147 (1989); L. J. Dixon, V. Kaplunovsky and J. Louis, "On Effective Field Theories Describing $(2,2)$ Vacua of the Heterotic String," Nucl. Phys. B 329, 27 (1990); J. P. Derendinger, C. Kounnas, P. M. Petropoulos and F. Zwirner, "Fluxes and gaugings: $\mathrm{N}=1$ effective superpotentials," Fortsch. Phys. 53, 926 (2005) arXiv:hep-th/0503229.

[14] E. Komatsu et al. [WMAP Collaboration], "Five-Year Wilkinson Microwave Anisotropy Probe (WMAP) Observations:Cosmological Interpretation," Astrophys. J. Suppl. 180, 330 (2009) arXiv:0803.0547 [astro-ph]].

[15] http://www.rssd.esa.int/Planck

[16] H. Murayama, H. Suzuki, T. Yanagida and J. Yokoyama, "Chaotic inflation and baryogenesis by right-handed sneutrinos," Phys. Rev. Lett. 70, 1912 (1993); J. R. Ellis, M. Raidal and T. Yanagida, "Sneutrino inflation in the light of WMAP: Reheating, leptogenesis and flavorviolating lepton decays," Phys. Lett. B 581 (2004) 9 arXiv:hep-ph/0303242.

[17] J. Giedt, G. L. Kane, P. Langacker and B. D. Nelson, "Massive neutrinos and (heterotic) string theory," Phys. Rev. D 71 (2005) 115013 arXiv:hep-th/0502032.

[18] S. Ferrara, C. Kounnas and F. Zwirner, "Mass formulae and natural hierarchy in string effective supergravities," Nucl. Phys. B 429, 589 (1994) [Erratum-ibid. B 433, 255 (1995)] arXiv:hep-th/9405188. R. Barbieri and S. Cecotti, "Radiative Corrections To The Effective Potential In N=1 Supergravity," Z. Phys. C 17 (1983) 183; M. K. Gaillard and V. Jain, "Supergravity coupled to chiral matter at one loop," Phys. Rev. D 49 (1994) 1951 arXiv:hep-th/9308090. 